\title{
Chronic bacteraemia due to Staphylococcus epidermidis after bone marrow transplantation
}

\author{
B. LINA* $\$$, F. FOREY*, J. D. TIGAUD $\dagger$ and J. FLEURETTE* \\ *Département de Recherche en Bactériologie Médicale (EA 1655), Faculté de Medécine Alexis Carrel, rue Guillaume \\ Paradin, 69372 Lyon Cedex 08 and † Service d'Hématologie, Pavillon E, Hôpital Edouard Herriot, 69437 Lyon Cedex \\ 03, France
}

\begin{abstract}
Summary. A chronic bacteraemia due to Staphylococcus epidermidis occurred in a patient undergoing allogeneic bone marrow transplantation. Forty-two S. epidermidis isolates were obtained from blood cultures over a period of 5 months. Isolates were separated into three groups by $S m a I$ macrorestriction characterisation with pulsed-field gel electrophoresis (PFE-1, one isolate; PFE-2, 32 isolates; PFE-3, nine isolates). Differences were detected in antimicrobial susceptibility patterns among isolates belonging to group PFE-2. The two strains, PFE-2 and PFE-3, were both responsible for the chronic bacteraemia and were isolated during different admissions to the hospital. A central venous catheter was the portal of entry for PFE-2. DNA macro-restriction analysis with pulsed-field gel electrophoresis was helpful in the epidemiological investigation of this $S$. epidermidis chronic bacteraemia.
\end{abstract}

\section{Introduction}

Coagulase-negative staphylococci (CNS) are the organisms most frequently isolated from blood cultures, and although the majority of isolations represent contamination, clinically important infections do occur and these organisms represent a major cause of septicaemia or severe diseases related to intra-vascular catheter colonisation ${ }^{1-3}$ or to severe immunodepression. ${ }^{4-6}$ Because of the ubiquitous nature of Staphylococcus epidermidis and of its pathogenic potential, strain characterisation of this organism has become increasingly important. ${ }^{7,8}$

The usual approach to determine the clinical relevance of $S$. epidermidis isolates is (i) to require repeated isolation from independent specimens from the patient, and (ii) that the isolates should share identical typing characteristics. Unfortunately, no single typing method is entirely satisfactory for $S$. epidermidis. ${ }^{9-11}$ Among recently developed epidemiological fingerprinting techniques, pulsed-field gel electrophoresis (PFGE) has been described as useful in strain characterisation of $S$. epidermidis, yielding stable and discriminatory restriction patterns. ${ }^{12-15}$

In this study, 42 isolates of $S$. epidermidis from 41

Received 3 March 1994; revised version accepted 29 Aug. 1994 $\dagger$ Present address: Laboratoire Central de Microbiologie, Bât. 10, Hôpital Edouard Herriot, Place d'Arsonval, 69437 Lyon Cedex 03, France. blood cultures performed over a 5-month period in a patient undergoing allogeneic bone marrow transplantation (BMT) were compared by analysis of the SmaI macro-restriction patterns in PFGE.

\section{Patient, materials and methods}

\section{Case report}

A 33-year-old man was admitted to the hospital for treatment of chronic myeloid leukaemia. After the course of chemotherapy, the patient received an allogeneic BMT from his HLA Dr-identical brother. After transplantation, two routine blood cultures were performed daily through the catheter. On day 10 , one blood culture yielded $S$. epidermidis, considered to be contaminant (antibiotic-resistance (AtB)-type A). Engraftment was achieved on day 17. The patient developed a chronic graft versus host disease (GVHD) on day 20. Between day 27 and day 55, 16 blood cultures were positive with S. epidermidis (AtB-type B). Isolates were from blood cultures performed through the catheter and in peripheral samples. Changes were observed in antimicrobial resistance of one isolate (AtB-type C). Despite intravenous vancomycin $(15 \mathrm{mg} / \mathrm{kg}$ daily) and oral pristinamycin (3 g daily), the patient remained febrile until the central venous line was removed on day 58. A S. epidermidis isolate with identical antibiogram to those of the blood cultures was obtained from this catheter (AtB-type B). 


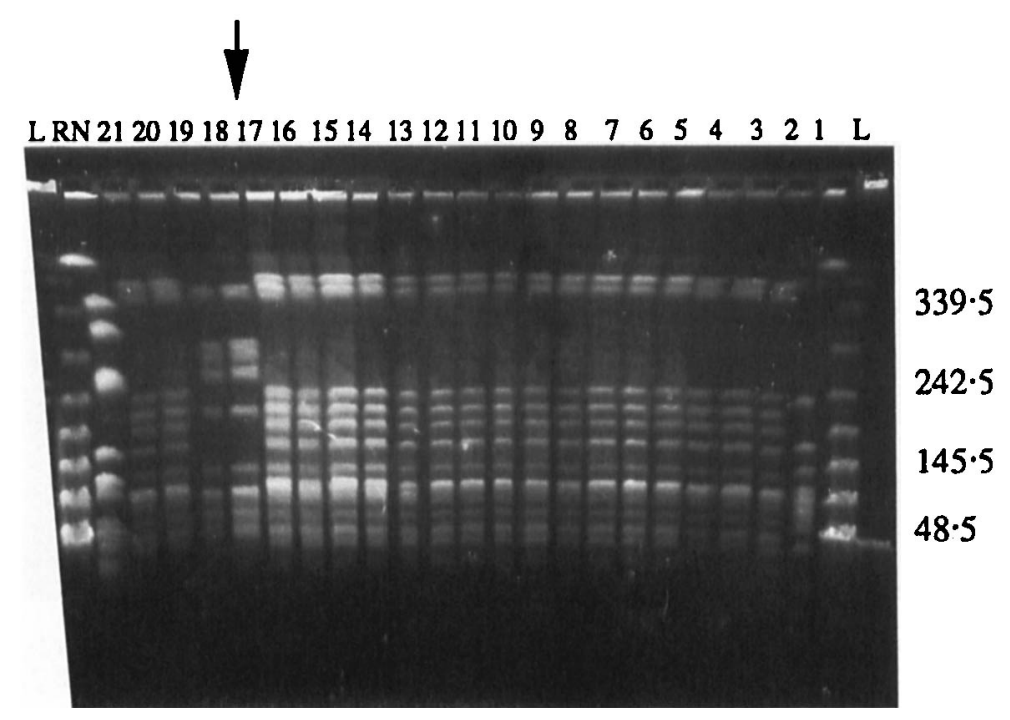

Fig. 1. SmaI pulsed-field patterns of the first 21 isolates (nos. 1-21). L, concatemers of phage $\lambda$ as molecular size markers (kb), RN, $S$. aureus RN4220, used as a control strain; $\rightarrow$ indicates catheter removal.

A second catheter was implanted and the antibiotic therapy was stopped. Of the 61 blood cultures performed through the second catheter before the first discharge, only three yielded $S$. epidermidis isolates, presenting different antimicrobial resistance patterns (AtB-types $\mathrm{C}$ and $\mathrm{E}$ ). The patient was discharged apyrexial on day 89 .

He was admitted to the hospital on day 115 with a macroscopic haematuria due to thrombocytopenia, and diarrhoea due to acute GVHD. He received platelet transfusion in association with high doses of methylprednisolone and oral vancomycin. On day 123, he developed fever and two blood cultures drawn through the catheter yielded $S$. epidermidis (AtB-types $B$ and E). Despite a course of intravenous vancomycin started on the same day $(15 \mathrm{mg} / \mathrm{kg}$ daily), both peripheral and catheter blood cultures were positive daily with $S$. epidermidis between days 135 and 144 (AtB-types B and D). Two vanco-locks were then performed through the catheter on day 145 , allowing the blood cultures to remain sterile for a week before re-appearance of $S$. epidermidis (AtB-types B and E). The catheter was removed on day 158 and the administration of intravenous vancomycin was discontinued. No $S$. epidermidis was isolated from the culture of the catheter nor from the blood-culture samples. The patient was discharged apyrexial.

\section{Bacterial strains and antibiotic susceptibility testing}

All strains were identified by the ID-32-Staph gallery (bioMérieux, Marcy-l'Etoile, France). Antimicrobial susceptibility was determined by the ATB-Staph gallery, as recommended by the manufacturer (bioMérieux) (table).

\section{Culture of the catheter tips}

The removed catheters were cultured according to the method described by Brun-Buisson et al ${ }^{16}$ Briefly, the catheter tip was immersed in $1 \mathrm{ml}$ of Brain Heart Infusion Broth (bioMérieux) and shaken vigorously for $1 \mathrm{~min}$. Then, $0 \cdot 1 \mathrm{ml}$ of the suspension was inoculated on to sheep blood agar (bioMérieux) allowing the number of cfu to be counted. Concomitantly, the catheter tip was cultured in the brain heart infusion broth.

\section{Chromosomal analysis by PFGE}

Chromosomal DNA was extracted as described previously. ${ }^{12}$ Intact DNA was digested with $30 \mathrm{U}$ of SmaI, as recommended by the manufacturer (Boehringer Mannheim, Meylan, France). PFGE was performed with a contour-clamped homogeneous electric field apparatus (CHEF-DR II; BioRad, Ivry Sur Seine, France) at $150 \mathrm{~V}$ for $20 \mathrm{~h}$ in cooled TBE buffer, with a constant pulse time of $20 \mathrm{~s}$. After the run, gels were stained for $15 \mathrm{~min}$ in ethidium bromide solution $10 \mathrm{mg} / \mathrm{ml}$ and photographed with a Polaroid camera under UV light.

\section{Results}

Three clearly different restriction pulsed patterns were observed (figs. 1 and 2). The first isolate (1) presented its own unique PFGE pattern (PFE-1) while the 41 remaining isolates were gathered into two groups of, respectively, 32 (group PFE-2) and nine isolates (group PFE-3); one blood culture yielded both strains (no. 37 in fig. 2).

Except for isolate no. 1, all isolates obtained before the first catheter was removed belonged to group PFE-2 (figs. 1 and 3). This strain was also isolated from the first catheter. After implantation of the second catheter and before discharge, the first two isolates belonged to group PFE-3 (figs. 1 and 3). Then, 


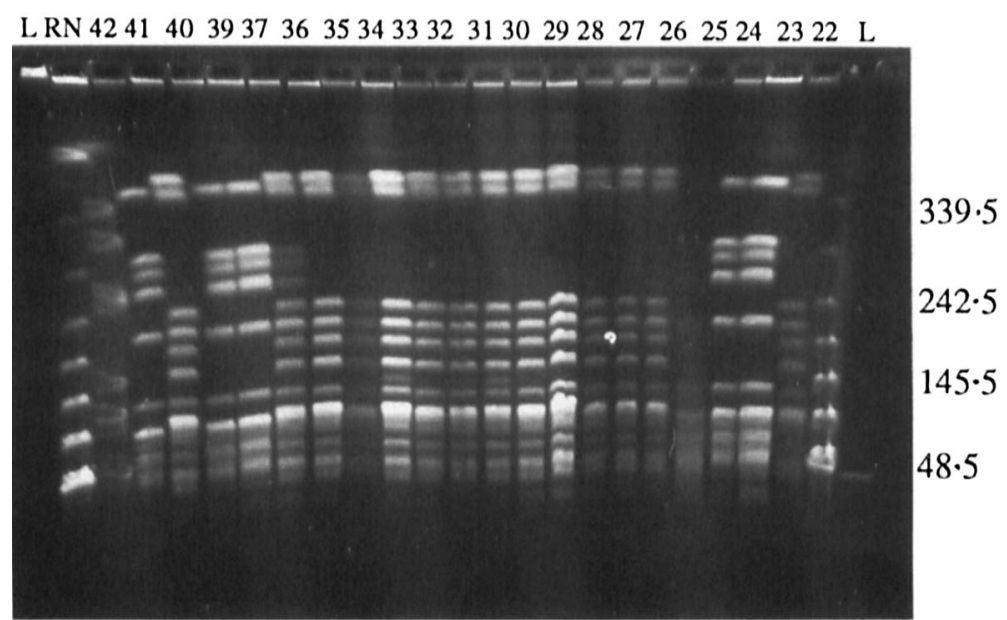

Fig. 2. SmaI pulsed-field patterns of isolates nos. 22-42. L, concatemers of phage $\lambda$ as molecular size marker (kb); RN, S. aureus RN4220, used as a control strain. Isolates from blood culture no. 37 displayed a combination of patterns PFE-2 and PFE-3; both strains were isolated from the blood sample.

Date

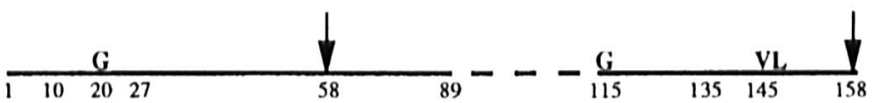

AtB-type $\left\{\begin{array}{l}\text { A } \\ B \\ C \\ D \\ E\end{array}\right.$

PFE-type $\left\{\begin{array}{l}1 \\ 2 \\ 3\end{array}\right.$ $\square$

प्र<smiles>[Te]=[Te]</smiles>

回

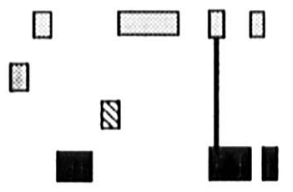

$\square$

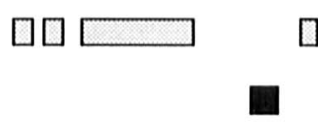

37

Fig. 3. Schematic representation of the correlation between AtB-types and PFE-types. AtB-B, C and D isolates shared the same PFE-Type (PFE-2). Blood culture no. 37 yielded both PFE-2 and -3 strains. Arrows indicate catheter removal; G, episodes of acute GVHD; VL, Vancolocks, -..-, discharge.

Table. Correlation between antibiotypes (AtB-types) and DNA restriction pulsed-field patterns (PFE-types) of the 42 S. epidermidis isolates

\begin{tabular}{cllc}
\hline Number of isolates & Antimicrobial resistance pattern & AtB-type & PFE-type \\
\hline 1 & pen & A & 1 \\
28 & pen/met/gen/pef/sxt & B & 2 \\
3 & pen/met/gen/ery/rif/pef/sxt/fos/fus & C & 2 \\
1 & pen/met/gen/pef/sxt/fos & D & 2 \\
9 & pen/met/gen/ery/prt/rif/pef/sxt/fos/fus & E & 3
\end{tabular}

Pen, penicillin; met, methicillin; gen, gentamicin; ery, erythromicin; prt, pristinamycin; rif, rifampicin; pef, pefloxacin; sxt, sulphamethoxazole + trimethoprim; fos, fosfomycin; fus, fusidic acid.

on the second admission to hospital, two PFE-2 isolates were obtained, followed by three PFE-3 isolates (figs. 2 and 3). Then PFE-2 isolates persisted until the vanco-locks were performed. Between the vanco-locks and the second catheter removal, PFE-2 and PFE-3 isolates were recovered alternately (figs. 2 and 3). "Isolate no. 37" displayed a pattern combining PFE-2 and PFE-3 patterns; both strains were isolated from the blood culture.

Changes were detected in antibiotic susceptibility of four isolates belonging to group PFE-2 (table). All PFE-3 isolates had the same antibiogram. 


\section{Discussion}

$S$. epidermidis is recognised as an important nosocomial pathogen in immunocompromised patients. The conventional approach to determine the clinical relevance of $S$. epidermidis isolates in a patient is to require repeated isolation from independent specimens of isolates that share typing characteristics. PFGE has been described as effective and discriminating in strain comparison, since it can provide easy-to-compare and unequivocal DNA restriction patterns. ${ }^{12-15}$

In the case described above, $S$. epidermidis isolates from blood cultures yielded three different pulsed field patterns, PFE-1, PFE-2 and PFE-3 (figs. 1 and 2). Types PFE-2 and PFE-3 gathered, respectively, 32 and nine isolates (blood culture no. 37 yielded both strains) with identical SmaI restriction patterns (figs. 1 and 2). Type PFE-2 isolates were obtained from blood samples before the first catheter was removed, from the first catheter tip, and from blood cultures after the catheter removal. Isolation from the culture of the catheter indicated that it was the portal of entry, but as the strain was isolated from blood cultures 28 days after the catheter removal, it is possible that the catheter was endogenously colonised from another site. One major reservoir of $S$. epidermidis is the gastrointestinal tract. ${ }^{17}$ The PFE-2 strain could have been present in the intestinal flora and been translocating chronically during the chronic intestinal GVHD developed in the patient. Unfortunately, $S$. epidermidis was not isolated from the faeces because the patient received an oral vancomycin regimen. A second explanation for this late recurrence may have been a possible metastatic localisation of the PFE-2 strain. There was no clinical evidence for any putative metastatic site, and except for the stool surveillance, no investigation was performed to assess its existence (for example, on skin, oral mucosa or catheter hub cultures).

\section{References}

1. Goldmann DA, Pier GB. Pathogenesis of infections related to intravascular catheterization. Clin Microbiol Rev 1993; 6: 176-192.

2. Dickinson GM, Bisno AL. Infections associated with indwelling devices: concepts of pathogenesis; infection associated with intravascular devices. Antimicrob Agents Chemother 1989; 33: 597-601.

3. Peters G. New considerations in the pathogenesis of coagulasenegative staphylococcal foreign body infections. $J$ Antimicrob Chemother 1988; 21 Suppl C: 139-148.

4. Peters G, Schumacher-Perdreau F, Pulverer G. Infections caused by coagulase-negative staphylococci in immunocompromised patients. Immun Infect 1986; 14: 165-169.

5. Wade JC, Schimpff SC, Newman KA, Wiernik PH. Staphylococcus epidermidis: an increasing cause of infection in patients with granulocytopenia. Ann Intern Med 1982; 97 : 503-508.

6. Winston DJ, Dudnick DV, Chapin M, Ho WG, Gale RP, Martin WJ. Coagulase-negative staphylococcal bacteraemia in patients receiving immunosuppressive therapy. Arch Intern Med 1983; 143: 32-36.
Changes in antimicrobial susceptibility were detected in four PFE-2 isolates (acquisition of resistance to fosfomycin in isolate no. 25 and to erythromycin, rifampicin, fosfomycin and fusidic acid in isolates nos. 7, 20 and 21). These changes lead the clinician to consider those isolates as different. Such switches in antibiotic susceptibility are known to occur in vivo, mostly due to the acquisition of resistance genes mediated by plasmids or transposons from reservoir strains. ${ }^{10}$ In the four observed variants, comparison of the restriction pulsed-field patterns demonstrated clonality of these variants with the other PFE-2 isolates. This emphasises the need for considerable care when assessing differences in isolates with minor differences in antimicrobial resistance patterns.

The pathogenic role of the second strain (PFE-3) was not clear. This pristinamycin-resistant strain may have emerged during the course of antimicrobial therapy and been responsible for bacteraemia during eradication of PFE-2 (fig. 3). However, as PFE-3 isolates were obtained during three short periods (fig. 3 ), this strain could also be considered as chronically contaminating blood cultures and not being of clinical relevance. No portal of entry was determined for PFE3. Nevertheless, as the blood samples were sterile after removal of the second catheter, it is likely that the catheter tip was the source of PFE-3, even if the strain was not isolated from the culture of this catheter. We assume that the vanco-locks were responsible for eradication of this PFE-3 strain from the catheter.

This observation emphasises that the pathogenic role of $S$. epidermidis remains difficult to analyse and that antimicrobial susceptibility patterns may lead clinicians to wrongly define two isolates as different. The use of PFGE will be of help in the understanding of both mechanisms of transmission and pathogenicity of coagulase-negative staphylococci, as well as in the epidemiological analysis of such recurrent bacteraemia.

7. Patrick CH, John JF, Levkoff AH, Atkins LM. Relatedness of strains of methicillin-resistant coagulase-negative Staphylococcus colonizing hospital personnel and producing bacteraemias in a neonatal intensive care unit. Pediatr Infect Dis $J$ 1992; 11: 935-940.

8. Renaud F, Freney J, Etienne $\mathbf{J}$ et al. Restriction endonuclease analysis of Staphylococcus epidermidis DNA may be a useful epidemiological marker. J Clin Microbiol 1988; 26: 1729-1734.

9. Dryden MS, Talsania HG, Martin S et al. Evaluation of methods for typing coagulase-negative staphylococci. $J$ Med Microbiol 1992; 37: 109-117.

10. Etienne $\mathbf{J}$, Renaud F, Bes $\mathbf{M}$ et al. Instability of characteristics amongst coagulase-negative staphylococci causing endocarditis. J Med Microbiol 1990; 32: 115-122.

11. Deighton M, Pearson S, Capstick J, Spelman D, Borland R. Phenotypic variation of Staphylococcus epidermidis isolated from a patient with native valve endocarditis. J Clin Microbiol 1992; 30: 2385-2390.

12. Lina B, Vandenesch F, Etienne J, Kreiswirth B, Fleurette J. Comparison of coagulase-negative staphylococci by pulsed-field gel electrophoresis. FEMS Microbiol Lett 1992; 92: 133-138.

13. Shayegani M, Parsons LM, Waring AL et al. Molecular 
relatedness of Staphylococcus epidermidis isolates obtained during a platelet transfusion-associated episode of sepsis. $J$ Clin Microbiol 1991: 29; 2768-2773.

14. Goering RV, Winters MA. Rapid method for epidemiological evaluation of gram-positive cocci by field inversion gel electrophoresis. J Clin Microbiol 1992; 30: 577-580.

15. Linhardt TF, Ziebuhr W, Meyer P, Witte W, Hacker J. Pulsedfield gel electrophoresis of genomic restriction fragments as a tool for the epidemiological analysis of Staphylococcus aureus and coagulase-negative staphylococci. FEMS Microbiol Lett 1992; 95: 181-186.

16. Brun-Buisson C, Abrouk F, Legrand P, Huet Y, Larabi S, Rapin M. Diagnosis of central venous catheter-related sepsis: Critical level of quantitative tip cultures. Arch Intern Med 1987; 147: 873-877.

17. Pfaller MA, Herwaldt LA. Laboratory, clinical, and epidemiological aspects of coagulase-negative staphylococci. Clin Microbiol Rev 1988; 1: 281-299. 